\title{
Controlling Self-Assembly Kinetics of DNA- Functionalized Liposomes Using Toehold Exchange Mechanism
}

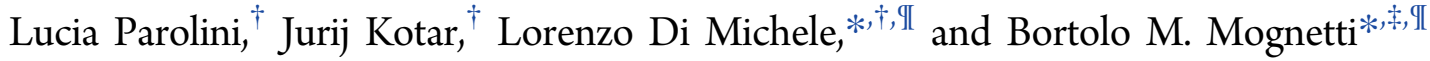

${ }^{\dagger}$ Biological and Soft Systems, Cavendish Laboratory, University of Cambridge, JJ Thomson Avenue, Cambridge CB3 0HE, United Kingdom

${ }^{\ddagger}$ Interdisciplinary Center for Nonlinear Phenomena and Complex Systems \& Service de Physique des Systémes Complexes et Mécanique Statistique, Université Libre de Bruxelles (ULB), Campus Plaine, CP 231, Blvd du Triomphe, B-1050 Brussels, Belgium

Supporting Information

ABSTRACT: The selectivity of Watson-Crick base pairing has allowed the design of DNA-based functional materials bearing an unprecedented level of accuracy. Examples include DNA origami, made of tiles assembling into arbitrarily complex shapes, and DNA coated particles featuring rich phase behaviors. Frequently, the realization of conceptual DNA-nanotechnology designs has been hampered by the lack of strategies for effectively controlling relaxations. In this article, we address the problem of kinetic control on DNA-mediated interactions between Brownian objects. We design a kinetic pathway based on toeholdexchange mechanisms that enables rearrangement of DNA bonds without the need for thermal denaturation, and test it on suspensions of DNA-functionalized liposomes, demonstrating tunability of aggregation rates over more than 1 order of magnitude. While the possibility to design complex phase behaviors using DNA as a glue is already well recognized, our results demonstrate control also over the kinetics of such systems.

KEYWORDS: DNA, kinetics, self-assembly, liposomes, toeholding, aggregation

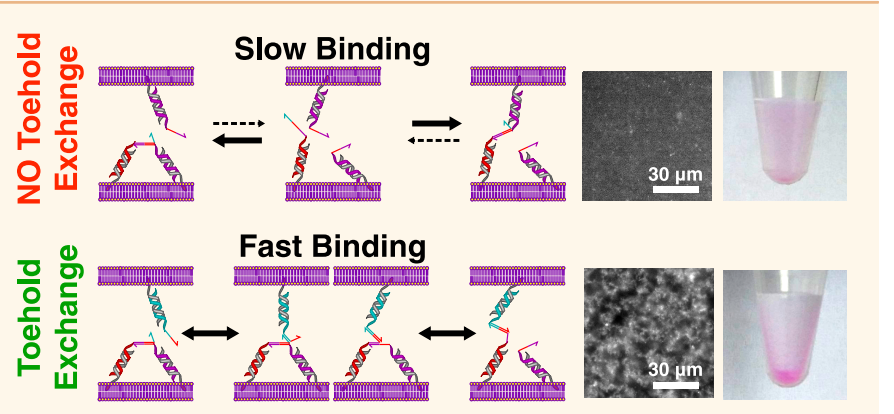

$\mathrm{D}$ NA nanotechnology has capitalized on the availability of a large library of oligomers that, in view of the selective nature of the Watson-Crick pairing, enable a large number of specific interactions to work simultaneously. ${ }^{2,3}$ In systems of DNA "bricks", this extreme selectivity makes it possible to design arbitrarily complex aggregates that selfassemble from short DNA strands with an unprecedented level of accuracy. ${ }^{4,5}$ The impact on engineering composite materials has been just as significant, with DNA-mediated interactions ${ }^{6-8}$ being used to design complex phase behaviors in DNA-coatedcolloid (DNACC) systems, ${ }^{9-12}$ to engineer ultrasensitive detectors $^{13,14}$ and microscopic walkers, ${ }^{15,16}$ or to create new biomimetic structures like DNA functionalized liposomes ${ }^{17-20}$ or artificial pores. ${ }^{21}$ These systems are striking examples of the potentialities offered by DNA to program bottom-up fabrication of functional materials.

So far, the use of tethered DNA to program colloidal selfassembly has focused on exploiting thermodynamics of DNA interactions rather than attempting to design kinetic DNADNA reactions. As a result, DNACC self-assembly has often been hampered by slow or in general poorly controlled relaxations, hindering equilibration. ${ }^{22,23}$
The straightforward approach to design kinetically accessible experiments consists of finely tuning environmental variables, particularly temperature, to a sweet spot where binding/ unbinding rates and interaction potentials are both suitable for reversible self-assembly. ${ }^{24,25}$ This strategy lacks flexibility and it is difficult to implement due to the high sensitivity of DNAmediated interactions to temperature changes, which narrows down the window of suitable experimental conditions. Only very recently, Wang et al. ${ }^{26}$ demonstrated that a high-density coating of weakly attractive DNA tethers facilitates equilibration over a broader range of temperatures. In these systems, however, kinetic behavior remains intimately connected to the thermodynamic ground state: neither can be changed without affecting the other.

Toehold-mediated strand-displacement mechanisms have been widely applied in DNA nanotechnology to enable isothermal control over the kinetics of DNA bonds. ${ }^{27-29}$ In the simplest implementation, a short single-stranded DNA

Received: November 15, 2015

Accepted: February 4, 2016

Published: February 4, 2016 
(ssDNA) domain, a toehold, transiently colocalizes an invading ssDNA molecule and a hybridized duplex. This catalyzes a branch migration reaction in which the invading strand displaces the incumbent strand from the duplex, releasing it in solution. If a second toehold catalyzes the inverse reaction, that is, the incumbent strand displacing back the invading strand, the scheme is known as Toehold Exchange Mechanism (TEM). ${ }^{30}$

To date, toeholding schemes have been applied to DNAmediated colloidal interactions to either enable structural rearrangements, ${ }^{31,32}$ or to engineer complex equilibrium phase diagrams. ${ }^{10}$ These schemes rely on adding free invading strands to the colloidal solution, which interfere or compete with bonds between DNA molecules tethered to the particles. At the supramolecular scale, the design theorized by Romano and Sciortino for systems of tetravalent DNA-nanostars enables direct bond swapping mediated by double-helical binders in solution. ${ }^{33}$

In this article, we introduce an interaction scheme based on TEM that enables direct control over the aggregation kinetics of colloidal units. We test our strategy on DNA-functionalized synthetic phospholipid vesicles (liposomes), often used to engineer responsive ${ }^{19}$ and biochemically active ${ }^{34}$ tissue-like materials, and in gene-delivery applications. ${ }^{14}$ As demonstrated in Figure 1, in our system liposome aggregation is hindered by a self-protected scheme, ${ }^{35}$ in which the formation of intraparticle loops is kinetically favored over interparticle bridges. The rate of loop opening and bridge formation limits the equilibration (Figure 1a) and is controlled by TEM $^{30}$ in which a transient three-strand state acts as a kinetic shortcut between inter- and intraparticle bonds (see Figure 1b,c). We show that the efficiency of the bond swapping depends on ligand stoichiometry, enabling aggregation-rate control over 1 order of magnitude.

In our scheme, invading strands are tethered to colloidal units (liposomes) and directly involved in bond formation, which eliminates the need for free linkers and enables bondswitching in one go. In contrast with existing strategies, our system allows to control the aggregation kinetics of the suspension almost independently from its equilibrium properties by tuning the relative stoichiometric concentrations of binders. This will result in a useful and generally applicable tool when designing bottom-up self-assembly.

\section{RESULTS AND DISCUSSION}

In Figure 1, we describe the system in more detail. We prepare Large Unilamellar lipid Vesicles (LUVs), $\approx 0.4 \mu \mathrm{m}$ in diameter, functionalized by four types of DNA linkers: $A_{1}, A_{2}, B$, and $I$. Constructs $A_{1}, A_{2}$, and $B$ are tipped by reactive sticky-end sequences, and feature a double-stranded (ds) DNA spacer tethered to the membrane by a double hydrophobic anchor. The latter includes a cholesterol and a cholesteryl molecule, and is irreversibly inserted within the fluid membrane, ${ }^{36}$ while enabling free lateral diffusion at a rate of a few squared micrometers per second $\left(\mu \mathrm{m}^{2} \mathrm{~s}^{-1}\right){ }^{37}$ Inert dsDNA strands $I$ (Figure 1b) do not carry any recognition sequence but act as repeller between approaching membranes, further slowing down loop-bridge swaps and extending the tunability range of the aggregation rate. We use spacers of contour length equal to 10 and $14 \mathrm{~nm}$ for the reactive and the inert constructs, respectively. LUVs are prepared from DOPC lipids doped with Texas Red DHPE for fluorescence imaging. See Methods section for details. The design of the sticky-end sequences
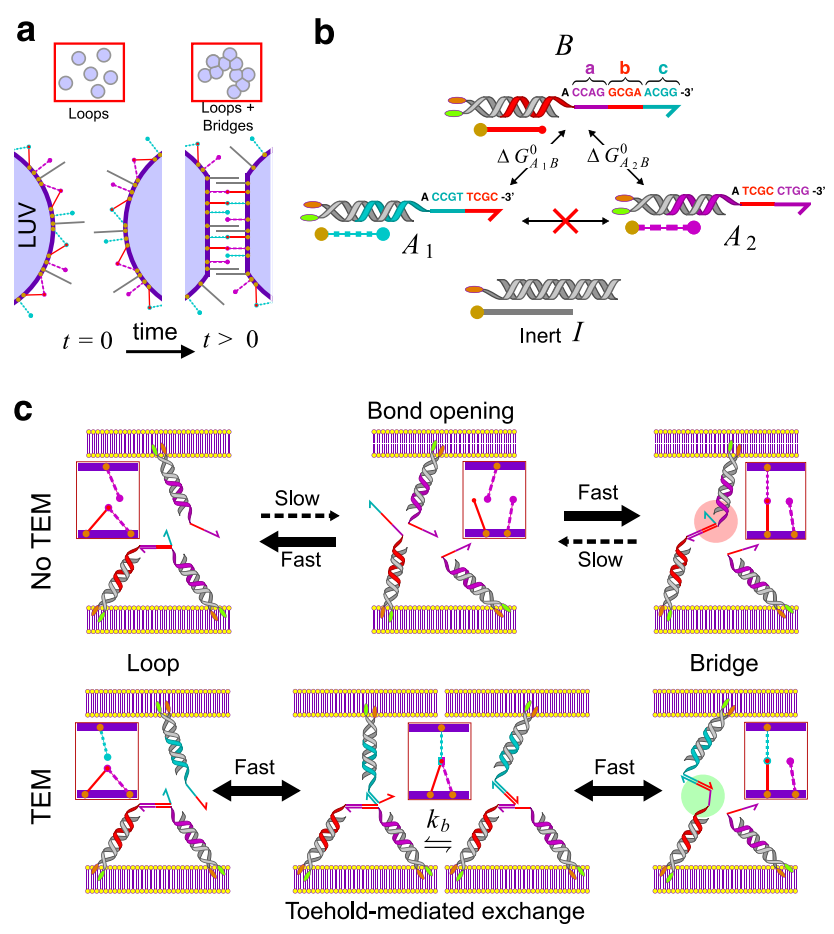

Figure 1. Microscopic ligand kinetics regulates vesicle aggregation. (a) Schematic view of the self-assembly process. After an initial quench $(t=0)$, intravesicle loops and steric repulsion due to inert strands slow down liposome aggregation. At $t>0$ aggregation is directed by bridge formation. (b) Structure and binding rules of DNA constructs. Each reactive tether $A_{1}, A_{2}$, and $B$, ends with a ssDNA sticky-end, made of two or three domains highlighted in different colors. The nonbinding A-base in black connects the sticky-ends to the dsDNA spacers. (c) Possible routes for loopbridge exchange: kinetically hindered mechanism involving thermal breakup of the loops (top) and toehold-exchange mechanism (bottom). In the right-hand side, we highlight the tail arrangement of the $A_{1} B$ (green) and of the $A_{2} B$ (red) bonds. Note that in the latter case domain $c$ of $B$ overlaps with the dsDNA spacer of $A_{2}$, an architecture expected to destabilize the link. ${ }^{1}$

follows the Toehold Exchange Mechanism of Zhang and Winfree. $^{30}$ In particular, sticky-end $B$ is composed of three domains: $a, b$, and $c$. Domain $b$ can bind both $A_{1}$ and $A_{2}$, while $a$ and $c$ (toeholding domains) exclusively bind $A_{2}$ and $A_{1}$, respectively. Because $A_{1}$ and $A_{2}$ can simultaneously bind $B$, the designed sequences catalyze the reaction

$$
A_{1}+A_{2} B \leftrightharpoons A_{1} B+A_{2}
$$

through the formation of $A_{1} A_{2} B$ complexes. The reaction in eq 1 drives the exchange of unbound tethers with those involved in stable bonds, mediating a swap between two different loops, two different bridges, and in particular between loops and bridges. The latter process significantly speeds up interparticle bond formation and thereby aggregation rate (Figure 1c).

Figure 2 demonstrates the effectiveness of the proposed scheme in controlling aggregation kinetics. For a fixed concentration of $B$ and $I$ strands, we prepare five different LUV suspensions in which we change the relative abundance of $A_{1}$ strands, $R=\left[A_{1}\right] /\left(\left[A_{1}\right]+\left[A_{2}\right]\right)$, while keeping $\left[A_{1}\right]+\left[A_{2}\right]$ $=2[B]$, where $[\ldots]$ indicates the number of DNA constructs per LUV. In our experiment $[B] \approx 360$ constructs/LUV. Changing the stoichiometry of $A$ strands enables control over TEM, disabling it for $R=0,1$ and maximizing its efficiency for $R=$ $1 / 2$. 

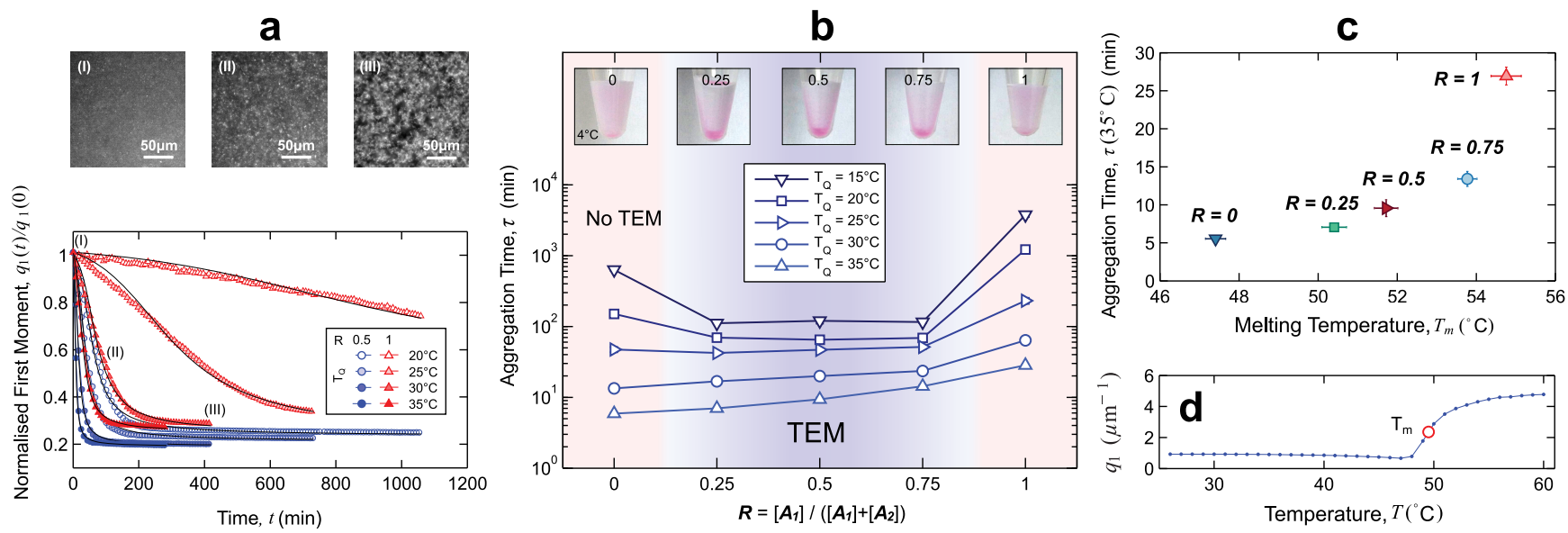

Figure 2. Toehold-mediated exchange speeds up liposome aggregation. (a) Aggregation kinetics of DNA-functionalized liposomes followed by the evolution of the normalized first moment of the structure factor. Blue circles and red triangles indicate samples with active $(R=1 / 2)$ and inactive $(R=1)$ TEM. Different shades indicate different quenching temperatures. Solid lines indicate fits with the empirical function reported in Methods. Top: epifluorescence snapshots of samples at early (left), intermediate (center), and late (right) aggregation stages. (b) Aggregation half-times for different coating stoichiometries and temperatures. Error bars are comparable with symbol size. Top: after 2 days at $4{ }^{\circ} \mathrm{C}$, samples featuring TEM exhibit aggregation and sedimentation. (c) Correlation between aggregation half-time at $35{ }^{\circ} \mathrm{C}$ and melting temperature $T_{\mathrm{m}}$. Different colors indicate various coating stoichiometries. (d) Melting curve for a $R=1 / 4$ sample used to measure the melting temperature $T_{\mathrm{m}}$.

The sequences, shown in Figure 1b, are designed to achieve similar hybridization free energies between $A_{1} / A_{2}$ and $\mathrm{B}\left(\Delta G_{A_{1} B}^{0}\right.$ $\left.\approx \Delta G_{A_{2} B}^{0}\right)$, as calculated using tabulated thermodynamic parameters of Watson-Crick base-pairing. ${ }^{38}$ Assuming that the relative concentrations of the three-strand complexes are negligible, this would result in five suspensions featuring the same equilibrium properties ${ }^{39,40}$ while displaying different kinetic behaviors. In experiments nonspecific effects bias $\Delta G_{A_{1} B}^{0}$ and $\Delta G_{A_{2} B}^{0}$ in different ways introducing a slight asymmetry between $A_{1}$ and $A_{2}$ strands as discussed below.

The suspensions are incubated at $60{ }^{\circ} \mathrm{C}$, above the melting temperature $T_{\mathrm{m}}$ of the LUV aggregates, then rapidly quenched to a temperature $T_{\mathrm{Q}}<T_{\mathrm{m}}$. The evolution of the system is then followed over time by capturing epifluorescence microscopy images, as shown in Figure 2a (top). Fourier analysis of the images enables the calculation of an approximate static structure factor $S(q ; t)$ as detailed in refs 41 and 42 . The function $S(q ; t)$, where $q$ is the spatial frequency and $t$ is the time, describes spatial density correlations: as a homogeneous suspension begins to aggregate, $S(q ; t)$ develops a peak at $q \approx$ $q_{\max }$, where $2 \pi / q_{\max }$ approximates the average scale of the aggregates. As the clusters coarsen, the peak grows in magnitude and shifts toward low- $q$ values. To keep track of the coarsening, we evaluate the first moment $q_{1}(t)=\int_{q<q^{*}}$ $q S(q ; t) / \int_{q<q^{*}} S(q ; t)$, with the cutoff $q^{*}=10 \mu \mathrm{m}^{-1}$. 33 The trajectories of $q_{1}(t) / q_{1}(0)$, where $t=0$ is the instant of the rapid quench, are then fitted with an empirical function (see Methods) to extract the aggregation half-time $\tau$ defined by $q_{1}(\tau) / q_{1}(0)=1 / 2$. Figure $2 \mathrm{a}$ shows the evolution of the first moment for samples with $R=1$ and $R=1 / 2$ quenched at different $T_{\mathrm{Q}}$. The rapid quench gives a strong kinetic advantage for the formation of intravesicle loops, effectively sequestrating nearly all of the $B$ strands available for bridge formation. ${ }^{35}$ Aggregation occurs when bridge population relaxes toward its nonzero equilibrium fraction. ${ }^{19}$ This process must follow one of the routes depicted in Figure 1c, namely the thermal breakup of loops (top) or the loop-bridge swap via TEM (bottom).
Although at $T_{\mathrm{Q}}=35{ }^{\circ} \mathrm{C}$ both samples aggregate at similar rates, at lower incubation temperatures, the sample with $R=1$ slows down significantly more than the one with $R=1 / 2$. This demonstrates that the thermal breakup of loops hinders bridge formation and that this kinetic bottleneck is bypassed for the system with $R=1 / 2$ by the kinetic shortcut established by TEM.

Figure $2 \mathrm{~b}$ summarizes the dependence of $\tau$ on $T_{\mathrm{Q}}$ and $R$, highlighting the strikingly different aggregation kinetics experienced at low $T_{\mathrm{Q}}$ by samples in which bridge formation is aided by TEM $(1 / 4 \leq R \leq 3 / 4)$, and those where TEM is inactive $(R=0,1)$. Images in the inset demonstrate the kinetic advantage of TEM samples at lower temperatures, hard to access with our microscopy setup. In particular, after incubating the samples for 2 days at $4{ }^{\circ} \mathrm{C}$, aggregation and sedimentation are observed almost only for $1 / 4 \leq R \leq 3 / 4$.

Aggregation times measured at high temperature (e.g., $T_{\mathrm{Q}}=$ $35{ }^{\circ} \mathrm{C}$ ) display a monotonic dependence on $R$, with samples featuring an excess of $A_{2}$ tethers aggregating faster than samples with more $A_{1}$. The asymmetry has a thermodynamic origin, as demonstrated in Figure $2 \mathrm{c}$ where we highlight the correlation between the aggregates' melting temperature $T_{\mathrm{m}}, R$, and $\tau$, measured at $T_{\mathrm{Q}}=35{ }^{\circ} \mathrm{C}$. The melting temperature of the aggregates is measured as the inflection point in $q_{1}(T)$ curves, collected upon slow heating of aggregated samples (Figure $2 \mathrm{~d}$ ). Figure $2 \mathrm{c}$ indicates that $A_{1} B$ bonds are more stable than $A_{2} B$ ones. Indeed, samples with large $R$ (i.e., $\left[A_{1}\right]>\left[A_{2}\right]$ ) display higher $T_{\mathrm{m}}$ caused by more stable bridges, and larger $\tau$ caused by slowly opening loops. The observed $A_{1}-A_{2}$ asymmetry is not reflected by the hybridization free-energies calculated accordingly to conventional nearest-neighbor thermodynamic rules, ${ }^{38,44-46}$ as detailed in the Supporting Information (SI) section S1.1. We argue that the experimental asymmetry arises from the recently reported inert-tail contributions to the hybridization free energy, ascribed to nonspecific Coulomb interactions. ${ }^{1}$ These particularly affect the $A_{2} B$ construct, where two tails, the dangling domain $c$ of sticky-end $B$ and the dsDNA spacer of $A_{2}$ emanate both from the same side of the hybridized 

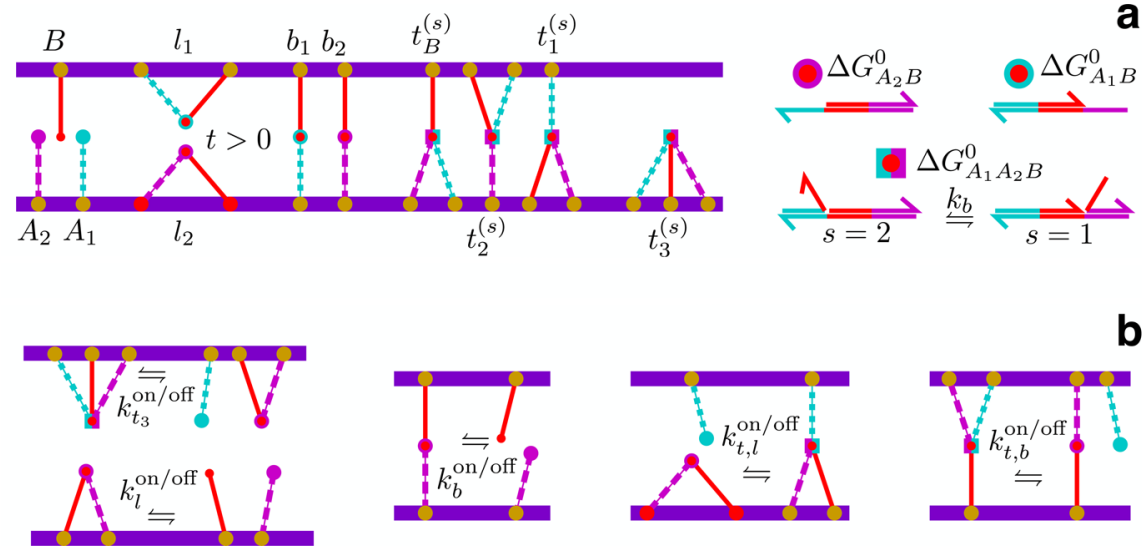

Figure 3. DNA complexes and binding-unbinding rates. (a) Possible single- two- and three-strand complexes formed by the system. In the case of three-strand complexes, the label $s$ indicates if $A_{1}(s=1)$ or $A_{2}(s=2)$ has invaded the middle domain of the sticky-end $B$ (top right and Figure 1). Branch migration takes place at a rate $k_{\mathrm{b}}$. (b) Binding/unbinding processes involving the complexes in panel a and respective reaction rate constants. Note that for isolated vesicles, only processes involving the formation of loops and $t_{3}$ complexes are possible, as shown in the left-most scheme. In the case of interacting vesicles, all processes are active.

duplex, as highlighted in Figure 1c (red circle). A similar arrangement has been shown to shift melting temperatures down by as much as $7{ }^{\circ} \mathrm{C}$. A geometry in which inert tails emanate from opposite sides of the duplex, as in $A_{1} B$ bonds (Figure 1c, green circle) has a smaller destabilizing effect. ${ }^{1}$ To test this hypothesis we measure the hybridization free energy of $A_{1} B$ and $A_{2} B$ duplexes with UV-absorbance spectroscopy, as detailed in SI section S1.1.1 and Table S1. Measurements carried out on the sticky-ends shown in Figure 1b, including or not the dangling " $\mathrm{A}$ " base, reveal only a minor difference between $\Delta G_{A_{1} B}^{0}$ and $\Delta G_{A_{2} B}^{0}$ that is insufficient to explain the trend in Figure $2 \mathrm{c}$. We then test sticky-ends featuring $T_{5}$ "tails" at their $5^{\prime}$ terminus, to partially mimic dsDNA spacers. In support of our hypothesis, we observe a substantial destabilization in the $A_{2} B$ duplex, where $T_{5}$ tail of $A_{2}$ emanates from the same side as the unbound $c$ domain of $B$ (see SI, Figures S1 and S2). Consistently, the free energy shift is much smaller in $A_{1} B$ where only one tail emanates from each side of the duplex. Remarkably, in samples capable of TEM, the observed thermodynamic asymmetry between $A_{1}$-rich and $A_{2}$ rich samples does not affect aggregation kinetics at low temperatures: for $T_{\mathrm{Q}}=15{ }^{\circ} \mathrm{C}$, samples with $1 / 4 \leq R \leq 3 / 4$ exhibit nearly identical aggregation times (Figure $2 \mathrm{~b}$ ). This indicates that at low temperature relaxation kinetics is fully controlled by TEM-mediated bond swaps, while the rate of thermal loop opening, affected by the asymmetry, becomes comparatively negligible. This evidence confirms the possibility of controlling kinetics independently from equilibrium thermodynamic properties.

We now demonstrate the correlation between experimental aggregation kinetics (Figure 2) and the typical time in which a population of loops relaxes to form bridges (Figure 1c), as calculated using a microscopic model. This will further confirm that the aggregation time is indeed limited by the thermal breakup of loops unless TEM is used. A complete derivation of the model is provided in the SI, sections S1 and S2.

Figure $3 \mathrm{a}$ describes the possible complexes formed by the tethers, which include two types of bridges and loops $\left(l_{1 / 2}\right.$ and $b_{1 / 2}$ engaging $A_{1}$ or $A_{2}$, respectively) and four types of threestrand complexes: $t_{B}, t_{1}, t_{2}$, and $t_{3}$. The latter is a complex made by two loops $\left(l_{1}\right.$ and $\left.l_{2}\right)$ sharing the same $B$ strand, while $t_{B}, t_{1}$, and $t_{2}$ are complexes in which $B, A_{1}$, or $A_{2}$ tethers, respectively, bind a complementary loop on an opposing vesicle.

We study the dynamics of bridge formation by approximating two interacting vesicles as two functionalized planes facing each other. To mimic the fact that loop population quickly equilibrates within each vesicle after the initial temperature quench, the initial $(t=0)$ concentrations of DNA complexes are chosen as for isolated vesicles at equilibrium, where only $l_{1 / 2}$ and $t_{3}$ complexes are possible. At the temperatures $T_{\mathrm{Q}}$ considered in Figure 2, which are well below the melting temperature of the DNA sticky-end dimerization, the population of loops is almost maximized $\left[l_{1}\right]+\left[l_{2}\right] \approx[B]$, while the fraction of $B$ strands involved in $t_{3}$ complexes is always below 5\% (see SI Figure S4). For $t>0$, we enable the formation of intervesicle complexes and track the total number of bridges as a function of time (Figure 4a). The time evolution of the concentration of different complexes has been obtained using mass balance kinetic equations (see SI section S2). Such equations are function of the reaction rates defined in Figure 3, in particular the binding/unbinding rates $k^{\text {on/off }}$ and the branchmigration rate $k_{\mathrm{b}}$. The latter describes the diffusive process through which domain $b$ of sticky-end $B$ migrates from $A_{1}$ to $A_{2}$ and vice versa, ${ }^{30}$ as demonstrated in Figure $1 \mathrm{c}$ and $3 \mathrm{a}$.

We assume that the off rates of tethered constructs $k^{\text {off }}$ are equal to those of the free strands in solution $k_{0}^{\text {off. }}$. This is a good approximation when the spacers do not hinder hybridization, ${ }^{47}$ and allows to calculate $k^{\text {off }}$ using the hybridization free energy $\Delta G^{0}$ and the dimerization rate $k_{0}^{\text {on }}$ of free sticky-ends in solution. For a generic complex, and taking $\rho_{0}=1 \mathrm{M}$ as the standard concentration, we obtain

$$
k^{\text {off }}=k_{0}^{\text {off }} \quad \frac{k_{0}^{\text {on }}}{k^{\text {off }}}=\frac{\exp \left[-\beta \Delta G^{0}\right]}{\rho_{0}} .
$$

In eq $2, \Delta G^{0}=\Delta G_{A_{1 / 2} B}^{0}$ for the reaction between $A_{1 / 2}$ and $B$ sticky-ends, while $\Delta G^{0}=\Delta G_{A_{1} A_{2} B}^{0}-\Delta G_{A_{1 / 2} B}^{0}$ for the reaction between $A_{1 / 2} B$ complexes and $A_{2 / 1}$. The hybridization free energy of each complex is calculated using nearest-neighbor thermodynamic rules ${ }^{38,44}$ as reported in SI section S1.1. The dimerization rate of short oligomers free in solution is typically estimated in $k_{0}^{\text {on }} \approx 10^{6} \mathrm{M}^{-1} \mathrm{~s}^{-1} \cdot{ }^{30,48,49}$ We do not attempt to refine such value based on the specific sequences used in our 

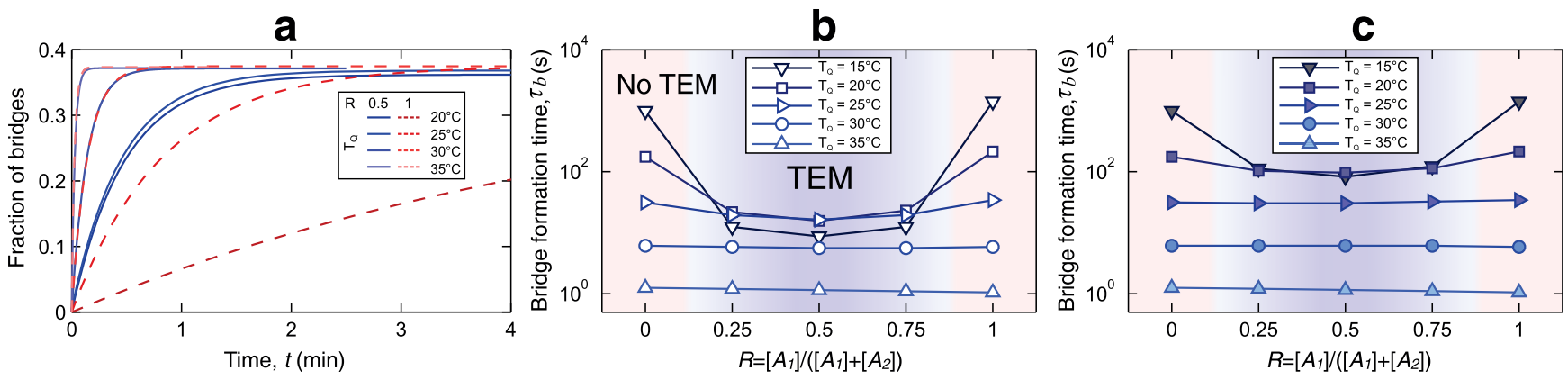

Figure 4. Theory shows a direct correlation between experimental aggregation and simulated bridge formation dynamics. (a) Relative concentration of bridges (normalized to the total number of $B$ tethers per vesicle) as a function of time starting from an initial configuration in which only loops are present. The stoichiometric ratio between $A_{1}$ and $A_{2}$ strands and the temperatures have been chosen as in Figure $2 a$. (b) Bridge formation half-times, defined as the times where the number of bridges is halfway between its initial and final value. We use the same conditions adopted in Figure $2 \mathrm{~b}$. The parameter $k_{\mathrm{b}}$ is chosen as $1 \mathrm{~s}^{-1}$. (c) Bridge formation half-times calculated with $k_{\mathrm{b}}=0.1 \mathrm{~s}^{-1}$ and the same conditions used in panel $b$.

experiments because the outcomes of our analysis are mainly biased by the uncertainties on $k_{\mathrm{b}}^{30,50}$ and on the hybridization free energy of the sticky-ends in the presence of inert-tails. ${ }^{1,50}$

Configurational costs hinder the hybridization of tethered constructs, as compared to free strands. ${ }^{19,39,40,51,52}$ Such contributions need to be included in the estimate of the on rates $k^{\text {on }}$, which differ from that of free sticky-ends $k_{0}^{\text {on }}$. In our previous studies, we estimated the configurational cost of loop and bridge formation in $1 / A L \rho_{0},{ }^{19,52}$ where $A$ is the area of the vesicle. In SI section S1.2, we generalize the calculation of the configurational costs to the three-strand complexes in Figure 3, obtaining the general expression

$$
\frac{k^{\text {on }}}{k^{\text {off }}}=\frac{\exp \left[-\beta \Delta G^{0}\right]}{\rho_{0} A L} \chi
$$

which allows to calculate $k^{\text {on }}$ from $k^{\text {off }}$ (eq 2). In eq 3, $\chi$ are geometric factors reported in SI section S1.2.

Equations 2 and 3 enable the calculation of reaction rates for all the processes shown in Figure $3 \mathrm{~b}$. In SI section S1.3, we list the values of the rates that have been used in this work.

Figure $4 \mathrm{a}$ shows the calculated time evolution of the total number of bridges in the same thermodynamic conditions of experiments shown in Figure 2a. After a transient stage, the number of bridges relaxes toward an equilibrium value that can be calculated using self-consistent theories ${ }^{39,40}$ that, in the present case, need to be generalized to include three-strand complexes. A detailed report will be presented elsewhere. We find that using TEM bridge formation time is reduced by 1 to 2 orders of magnitude at low temperature, while it remains unaffected at high temperature.

A summary of the calculated bridge-formation half-times $\tau_{\mathrm{b}}$ is shown in Figure $4 \mathrm{~b}$ as a function of $R$ and $T_{\mathrm{Q}}$. We recover the same trends observed in Figure $2 \mathrm{~b}$ for experimental aggregation times. This qualitative match confirms the direct correlation between the engineered nanoscale reactions and the macroscopic response of the system and, in particular, demonstrates that the mechanism responsible of the kinetic acceleration in TEM samples is indeed the formation of three-strand complexes $\left(t_{B}, t_{1}\right.$, and $t_{2}$ in Figure 3$)$ catalyzing loop-bridge swaps (Figure 1c). Although predicted bridge-formation times and experimental aggregation times follow similar trends as a function of $T_{\mathrm{Q}}$ and $R$, they are different in magnitude. This is expected as our model focuses on the microscopic kinetics of
DNA reactions and does not account for the Brownian diffusion of the LUVs.

Moreover, the magnitude of the equilibration rates shown in Figure 4 strongly depends on the poorly understood parameter $k_{\mathrm{b}}$ (Figures 1 and 3 ). The data in Figure $4 \mathrm{a}, \mathrm{b}$ are obtained using $k_{\mathrm{b}}=1 \mathrm{~s}^{-1}$, as proposed by Zhang and Winfree. ${ }^{30}$ This estimate has been recently questioned by Srinivas et al.; ${ }^{50}$ therefore, in Figure $4 \mathrm{c}$ we report results obtained with $k_{\mathrm{b}}=0.1 \mathrm{~s}^{-1}$. As expected, a reduction in $k_{\mathrm{b}}$ results in slower equilibration in TEM samples.

The experimental difference between the binding strength of $A_{1} B$ and $A_{2} B$ duplexes, as mentioned above, caused by inert-tail effects, is not captured by state-of-art thermodynamic models. In view of the absence of an exhaustive parametrization of inerttail effects, we calculate the hybridization free energies for our theoretical model using conventional nearest-neighbor rules. ${ }^{38,44-46}$ In doing so, we neglect the attractive effect of dangling bases, expectedly overcompensated by repulsive tail effects. ${ }^{1}$ Similarly, Srinivas et al. ${ }^{50}$ recently questioned the occurrence of coaxial stacking at the nick-site during the branch migration process; thus, we do not include stacking terms in the estimate of $\Delta G_{A_{1} A_{2} B}$. A comprehensive discussion on free energy estimates produced by different models and parametrizations, including an assessment of the effects dangling terms and coaxial stacking, is presented in the SI, section S1.1 and Figure S3.

Finally, we discuss possible design variations and constraint in our interaction scheme. Strand displacement rates increase exponentially with toehold length (or binding strength), saturating when the overhangs reach $\sim 6$ bases. ${ }^{30}$ Our choice of adopting 4-base toeholds is motivated by the need of limiting the fraction of three-strand complexes, which would increase if $a$ and $c$ domains were extended. A small fraction of three-strand complexes is required to guarantee that the equilibrium free energy of the system is weakly dependent on $R$, as changing the $A_{1}$ to $A_{2}$ ratio necessarily affects the concentration of $A_{1} A_{2} B$ complexes. Moreover, a large enough number of intervesicle three-strand complexes could, in principle, induce vesicle aggregation without the need of forming bridges. Although with the current design these scenarios are ruled out by theoretical analysis and control experiments (see SI section S3), we argue that longer toeholds may not be suitable (see Figure S5). 


\section{CONCLUSIONS}

In this article, we propose a new mechanism to control the kinetics of DNA mediated interactions. This is tested on liposomes that are functionalized with three different types of DNA thethers, two of which $\left(A_{1}\right.$ and $\left.A_{2}\right)$ compete to bind the third $(B)$. Upon rapid cooling intravesicle loops saturate all the available $B$ tethers, slowing down aggregation. The formation of intervesicle bridges is catalyzed by a toeholding exchange mechanism involving three-strand complexes $A_{1} A_{2} B .^{28,30}$ Experiments demonstrate that aggregation kinetics can be controlled over 1 order of magnitude by changing the relative stoichiometric concentration of $A_{1}$ and $A_{2}$. A new theoretical framework for the description of strand-exchange mechanism of tethered binders confirms the role played by the three-strand reactions in mediating bridge formation.

The proposed mechanism is general and can readily find application in other systems that use DNA as a glue to direct interactions.

Although we did not test our scheme for the case of binders with fixed anchoring points, we argue that the lateral diffusivity of the DNA on the lipid membrane may be key to facilitate three-strand reactions. Nonetheless, recently introduced functionalization procedures enabling a large increase in binders surface density on solid particles ${ }^{26}$ will certainly be useful when applying the mechanism proposed here.

\section{METHODS}

Large unilamellar vesicles of DOPC (Avanti Polar Lipids) are produced by extrusion in $300 \mathrm{mM}$ sucrose solution (Sigma-Aldrich) using a hand-driven mini-extruder (Avanti Polar Lipids) with a polycarbonate track-etched membrane $\left(400 \mathrm{~nm}\right.$ pores, Whatman) ${ }^{52}$ Vesicles are doped with $0.8 \%$ molar fraction of DHPE Texas red (Molecular Probes) to enable fluorescence imaging. Total lipid concentration in the extruded solution is $12.7 \mathrm{mM}$. DNA constructs are prehybridized as detailed in refs 19 and 52 from single-stranded DNA sequences. Sequences for reactive constructs $A_{1}, A_{2}$, and $B$ are (i) 5'-CGT GCG CTG GCG TCT GAA AGT CGA TTG CGA AAA- $3^{\prime}$ [Cholesterol TEG], (ii) [Cholesteryl TEG] 5'-TTT TCG CAA TCG ACT TT-3', and (iii) 5'-C AGA CGC CAG CGC ACG A [stickyend]-3', where sticky-end sequences are shown in Figure $1 \mathrm{~b}$. Sequences for inert construct $I$ are (j) 5'-CTA CTA TGG CGG GTG ATA AAA AAC GGG AAG AGC ATG CCC ATC CAA AA-3' [Cholesterol TEG] and (ji) 5'-GGA TGG GCA TGC TCT TCC CGT TTT TTA TCA CCC GCC ATA GTA GA-3'. Strands (i), (iii), (j), and (ij) are purchased from Integrated DNA Technologies; strand (ii) is purchased from Eurgentec. Since active constructs are composed of three ssDNA units, a nick is present in the resulting dsDNA spacer. Samples are prepared by mixing $10 \mu \mathrm{L}$ of extruded vesicle solution with $90 \mu \mathrm{L}$ of iso-osmolar solution containing $87 \mathrm{mM}$ glucose (SigmaAldrich), $100 \mathrm{mM} \mathrm{NaCl}$ (Sigma-Aldrich), TE buffer (Tris-EDTA, Sigma-Aldrich) and DNA constructs. In all samples, the final bulk DNA concentration is $320 \mathrm{nM}$ for $B, 640 \mathrm{nM}$ for $A_{1}+A_{2}$, and $800 \mathrm{nM}$ for $I$ constructs. Samples are injected into flat borosilicate glass capillaries $\left(4 \times 0.2 \mathrm{~mm}^{2}\right.$ internal section, CM Scientific), protected on both ends with a droplet of mineral oil (Sigma-Aldrich) and permanently sealed with epoxy glue (Araldite). Aggregation experiments are carried out on a fully automated Nikon Eclipse Ti-E inverted microscope using a Nikon PLAN APO $20 \times 0.75$ N.A. dry objective and a IIDC Point Gray Research Grasshopper-3 GS3-U323S6M-C camera. Five sample capillaries corresponding to the coating stoichiometries shown in Figure $2 \mathrm{~b}$ are lined up on a copper plate connected to a tailor-made Peltier stage that enables programmable temperature cycling. The microscope is equipped with a Perfect Focusing System (Nikon) to correct for vertical thermal drift. Samples are incubated at $60{ }^{\circ} \mathrm{C}$ for $50 \mathrm{~min}$ to enable homogenization, then quenched to $T_{\mathrm{Q}}$ and imaged to track aggregation. Using a programmable motorized stage, we loop over all the samples. Fourier analysis of the epifluorescence images is carried out as explained in the main text and literature. ${ }^{41,42}$ Normalized first moment $q_{1}(t) / q_{1}(0)$, shown in Figure 2a, is fitted with the empirical function

$$
f(t)=\frac{1-\alpha}{2}\left[1-\frac{t-\tau}{\sqrt{(t-\tau)^{2}+\sigma \tau t}}\right]+\alpha
$$

where the parameter $\sigma$ has been fixed to $0.77, \tau$ is the aggregation halftime, and $\alpha$ is the plateau value reached at $t \rightarrow \infty$.

\section{ASSOCIATED CONTENT}

\section{S Supporting Information}

The Supporting Information is available free of charge on the ACS Publications website at DOI: 10.1021/acsnano.5b07201.

Full details on the derivation of the kinetic model and the thermodynamic parameters of DNA hybridization (PDF)

\section{AUTHOR INFORMATION}

\section{Corresponding Authors}

*E-mail: bmognett@ulb.ac.be.

*E-mail: 1d389@cam.ac.uk.

\section{Author Contributions}

${ }^{\mathrm{II}}$ L.D.M. and B.M.M. contributed equally.

\section{Notes}

The authors declare no competing financial interest.

\section{ACKNOWLEDGMENTS}

The authors thank P. Cicuta for scientific insight and proofreading the manuscript. L.P., L.D.M. and J.K. acknowledge support from the EPRSC Programme Grant CAPITALS number EP/J017566/1. L.D.M. acknowledges support from the Oppenheimer Fund and Emmanuel College Cambridge. B.M.M. acknowledges support from the Univeriste Libre de Bruxelles (ULB). In compliance with the requirements of EPSRC, a complete experimental dataset is available for download at https://www.repository.cam.ac.uk/handle/1810/ 253615.

\section{REFERENCES}

(1) Di Michele, L.; Mognetti, B. M.; Yanagishima, T.; Varilly, P.; Ruff, Z.; Frenkel, D.; Eiser, E. Effect of Inert Tails on the Thermodynamics of DNA Hybridization. J. Am. Chem. Soc. 2014, $136,6538-6541$

(2) Seeman, N. C. Structural DNA Nanotechnology: Growing Along with Nano Letters. Nano Lett. 2010, 10, 1971-1978.

(3) Jones, M. R.; Seeman, N. C.; Mirkin, C. A. Programmable Materials and the Nature of the DNA Bond. Science 2015, 347, 1260901.

(4) Ke, Y.; Ong, L. L.; Shih, W. M.; Yin, P. Three-Dimensional Structures Self-Assembled from DNA Bricks. Science 2012, 338, 11771183.

(5) Mohammed, A. M.; Schulman, R. Directing Self-Assembly of DNA Nanotubes Using Programmable Seeds. Nano Lett. 2013, 13, 4006-4013.

(6) Alivisatos, A. P.; Johnsson, K. P.; Peng, X.; Wilson, T. E.; Loweth, C. J.; Bruchez, M. P.; Schultz, P. G. Organization of 'Nanocrystal Molecules' Using DNA. Nature 1996, 382, 609.

(7) Mirkin, C. A.; Letsinger, R. C.; Mucic, R. C.; Storhoff, J. J. A DNA-Based Method for Rationally Assembling Nanoparticles into Macroscopic Materials. Nature 1996, 382, 607.

(8) Di Michele, L.; Eiser, E. Developments in Understanding and Controlling Self Assembly of DNA-Functionalized Colloids. Phys. Chem. Chem. Phys. 2013, 15, 3115-3129. 
(9) Angioletti-Uberti, S.; Mognetti, B. M.; Frenkel, D. Reentrant Melting as a Design Principle for DNA-Coated Colloids. Nat. Mater. 2012, 11, 518-522.

(10) Rogers, W. B.; Manoharan, V. N. Programming Colloidal Phase Transitions with DNA Strand Displacement. Science 2015, 347, 639642.

(11) Wang, Y.; Wang, Y.; R, D.; Manoharan, V. N.; Feng, L.; Hollingsworth, A. D.; Weck, M.; Pine, D. J. Colloids with Valence and Specific Directional Bonding. Nature 2012, 491, 51-55.

(12) Halverson, J. D.; Tkachenko, A. V. DNA-Programmed Mesoscopic Architecture. Phys. Rev. E 2013, 87, 062310.

(13) Taton, T. A.; Mirkin, C. A.; Letsinger, R. L. Scanometric DNA Array Detection with Nanoparticle Probes. Science 2000, 289, 17571760 .

(14) Banga, R. J.; Chernyak, N.; Narayan, S. P.; Nguyen, S. T.; Mirkin, C. A. Liposomal Spherical Nucleic Acids. J. Am. Chem. Soc. 2014, 136, 9866-9869.

(15) Muscat, R. A.; Bath, J.; Turberfield, A. J. A Programmable Molecular Robot. Nano Lett. 2011, 11, 982-987.

(16) Martinez Veracoechea, F.; Mognetti, B. M.; Angioletti-Uberti, S.; Varilly, P.; Frenkel, D.; Dobnikar, J. Designing Stimulus-Sensitive Colloidal Walkers. Soft Matter 2014, 10, 3463-3470.

(17) Beales, P. A.; Vanderlick, T. K. Application of Nucleic AcidLipid Conjugates for the Programmable Organisation of Liposomal Modules. Adv. Colloid Interface Sci. 2014, 207, 290-305.

(18) Beales, P. A.; Vanderlick, T. K. Specific Binding of Different Vesicle Populations by the Hybridization of Membrane-Anchored DNA. J. Phys. Chem. A 2007, 111, 12372-12380.

(19) Parolini, L.; Mognetti, B. M.; Kotar, J.; Eiser, E.; Cicuta, P.; Di Michele, L. Volume and Porosity Thermal Regulation in Lipid Mesophases by Coupling Mobile Ligands to Soft Membranes. Nat. Commun. 2015, 6, 5948.

(20) van Lengerich, B.; Rawle, R. J.; Bendix, P. M.; Boxer, S. G. Individual Vesicle Fusion Events Mediated by Lipid-Anchored DNA. Biophys. J. 2013, 105, 409-419.

(21) Göpfrich, K.; Zettl, T.; Meijering, A. E. C.; Hernández-Ainsa, S.; Kocabey, S.; Liedl, T.; Keyser, U. F. DNA-Tile Structures Induce Ionic Currents through Lipid Membranes. Nano Lett. 2015, 15, 3134-3138.

(22) Dreyfus, R.; Leunissen, M. E.; Sha, R.; Tkachenko, A.; Seeman, N. C.; Pine, D. J.; Chaikin, P. M. Aggregation-Disaggregation Transition of DNA-Coated Colloids: Experiments and Theory. Phys. Rev. E 2010, 81, 041404.

(23) Mognetti, B. M.; Leunissen, M. E.; Frenkel, D. Controlling the Temperature Sensitivity of DNA-Mediated Colloidal Interactions Through Competing Linkages. Soft Matter 2012, 8, 2213.

(24) Nykypanchuk, D.; Maye, M. M.; van der Lelie, D.; Gang, O. DNA-guided Crystallization of Colloidal Nanoparticles. Nature 2008, $451,549-552$

(25) Kim, A. J.; Biancaniello, P. L.; Crocker, J. C. Engineering DNAMediated Colloidal Crystallization. Langmuir 2006, 22, 1991-2001.

(26) Wang, Y.; Wang, Y.; Zheng, X.; Ducrot, É.; Yodh, J. S.; Weck, M.; Pine, D. J. Crystallization of DNA-Coated Colloids. Nat. Commun. 2015, 6, 7253.

(27) Zhang, D. Y.; Turberfield, A. J.; Yurke, B.; Winfree, E. Engineering Entropy-Driven Reactions and Networks Catalyzed by DNA. Science 2007, 318, 1121-1125.

(28) Yurke, B.; Turberfield, A. J.; Mills, A. P.; Simmel, F. C.; Neumann, J. L. A DNA-Fuelled Molecular Machine Made of DNA. Nature 2000, 406, 605-608.

(29) Zhang, D. Y.; Seelig, G. Dynamic DNA Nanotechnology Using Strand-Displacement Reactions. Nat. Chem. 2011, 3, 103-113.

(30) Zhang, D. Y.; Winfree, E. Control of DNA Strand Displacement Kinetics Using Toehold Exchange. J. Am. Chem. Soc. 2009, 131, 17303-17314.

(31) Maye, M. M.; Kumara, M. T.; Nykypanchuk, D.; Sherman, W. B.; Gang, O. Switching Binary States of Nanoparticle Superlattices and Dimer Clusters by DNA Strands. Nat. Nanotechnol. 2010, 5, 116-120.

(32) Tison, C. K.; Milam, V. T. Reversing DNA-Mediated Adhesion at a Fixed Temperature. Langmuir 2007, 23, 9728-9736.
(33) Romano, F.; Sciortino, F. Switching Bonds in a DNA Gel: an All-DNA Vitrimer. Phys. Rev. Lett. 2015, 114, 078104.

(34) Hadorn, M.; Boenzli, E.; Sørensen, K. T.; De Lucrezia, D.; Hanczyc, M. M.; Yomo, T. Defined DNA-Mediated Assemblies of Gene-Expressing Giant Unilamellar Vesicles. Langmuir 2013, 29, 15309-15319.

(35) Leunissen, M. E.; Dreyfus, R.; Cheong, F. C.; Grier, D. G.; Sha, R.; Seeman, N. C.; Chaikin, P. M. Switchable Self-Protected Attractions in DNA-Functionalized Colloids. Nat. Mater. 2009, 8, 590-595.

(36) Pfeiffer, I.; Höök, F. Bivalent Cholesterol-Based Coupling of Oligonucletides to Lipid Membrane Assemblies. J. Am. Chem. Soc. 2004, 126, 10224-10225.

(37) van der Meulen, S. A. J.; Leunissen, M. E. Solid Colloids with Surface-Mobile DNA Linkers. J. Am. Chem. Soc. 2013, 135, 1512915134.

(38) SantaLucia, J. A Unified View of Polymer, Dumbbell, and Oligonucleotide DNA Nearest-Neighbor Thermodynamics. Proc. Natl. Acad. Sci. U. S. A. 1998, 95, 1460-1465.

(39) Varilly, P.; Angioletti-Uberti, S.; Mognetti, B. M.; Frenkel, D. A General Theory of DNA-Mediated and Other Valence-Limited Colloidal Interactions. J. Chem. Phys. 2012, 137, 094108-094122.

(40) Angioletti-Uberti, S.; Varilly, P.; Mognetti, B. M.; Tkachenko, A.; Frenkel, D. Communication: A Simple Analytical Formula for the Free Energy of Ligand-Receptor-Mediated Interactions. J. Chem. Phys. 2013, 138, 021102-021106.

(41) Di Michele, L.; Fiocco, D.; Varrato, F.; Sastry, S.; Eiser, E.; Foffi, G. Aggregation Dynamics, Structure, and Mechanical Properties of Bigels. Soft Matter 2014, 10, 3633-3648.

(42) Di Michele, L.; Varrato, F.; Kotar, J.; Nathan, S. H.; Foffi, G.; Eiser, E. Multistep Kinetic Self-Assembly of DNA-Coated Colloids. Nat. Commun. 2013, 4, 2007.

(43) Foffi, G.; De Michele, C.; Sciortino, F.; Tartaglia, P. Arrested Phase Separation in a Short-Ranged Attractive Colloidal System: a Numerical Study. J. Chem. Phys. 2005, 122, 224903.

(44) SantaLucia, J., Jr; Hicks, D. The Thermodynamics of DNA Structural Motifs. Annu. Rev. Biophys. Biomol. Struct. 2004, 33, 415440 .

(45) Markham, N. R.; Zuker, M. DINAMelt Web Server for Nucleic Acid Melting Prediction. Nucleic Acids Res. 2005, 33, W577-W581.

(46) Zadeh, J. N.; Steenberg, C. D.; Bois, J. S.; Wolfe, B. R.; Pierce, M. B.; Khan, A. R.; Dirks, R. M.; Pierce, N. A. NUPACK: Analysis and Design of Nucleic Acid Systems. J. Comput. Chem. 2011, 32, 170-173.

(47) Ho, D.; Zimmermann, J. L.; Dehmelt, F. A.; Steinbach, U.; Erdmann, M.; Severin, P.; Falter, K.; Gaub, H. E. Force-Driven Separation of Short Double-Stranded DNA. Biophys. J. 2009, 97, $3158-3167$.

(48) Morrison, L. E.; Stols, L. M. Sensitive Fluorescence-Based Thermodynamic and Kinetic Measurements of DNA Hybridization in Solution. Biochemistry 1993, 32, 3095-3104.

(49) Wetmur, J. G.; Davidson, N. Kinetics of Renaturation of DNA. J. Mol. Biol. 1968, 31, 349-370.

(50) Srinivas, N.; Ouldridge, T. E.; Šulc, P.; Schaeffer, J. M.; Yurke, B.; Louis, A. A.; Doye, J. P.; Winfree, E. On the Biophysics and Kinetics of Toehold-Mediated DNA Strand Displacement. Nucleic Acids Res. 2013, 41, 10641-10658.

(51) Angioletti-Uberti, S.; Varilly, P.; Mognetti, B. M.; Frenkel, D. Mobile Linkers on DNA-Coated Colloids: Valency without Patches. Phys. Rev. Lett. 2014, 113, 128303-128306.

(52) Shimobayashi, S.; Mognetti, B. M.; Parolini, L.; Orsi, D.; Cicuta, P.; Di Michele, L. Direct Measurement of DNA-Mediated Adhesion Between Lipid Bilayers. Phys. Chem. Chem. Phys. 2015, 17, 15615. 